\title{
Trombofilia hereditaria y trombo intracardíaco
}

\section{José Miguel Torres-Martel ${ }^{a, *}$ y Karla Monserrat Bautista-Hernández ${ }^{\mathrm{b}}$}

\author{
a Departamento de Cardiología Pediátrica, Hospital General Regional No. 251, Instituto Mexicano del Seguro Social, Metepec, \\ Estado de México, México \\ ${ }^{\mathrm{b}}$ Facultad de Medicina, Universidad Autónoma de Querétaro, Querétaro, México
}

Recibido el 7 de diciembre de 2016; aceptado el 24 de octubre de 2017

Disponible en Internet el 7 de abril de 2018

\section{PALABRAS CLAVE \\ Trombosis; \\ Ecocardiografía; \\ Tratamiento \\ anticoagulante}

\section{KEYWORDS}

Thrombosis;

Echocardiography;

Anticoagulant

treatment

\begin{abstract}
Resumen La trombofilia es una condición hematológica que predispone a eventos tromboembólicos venosos y arteriales. Es un factor que predispone a trombos intracardíacos en la edad pediátrica y se debe sospechar si no hay otros factores que predispongan a trombosis. Se reporta el caso de una paciente con antecedente de trombosis venosa cerebral a quien se le diagnosticó trombofilia por mutación homocigota G202010A del gen de la protrombina. Desarrolló un trombo auricular derecho, el cual fue tratado con anticoagulación y vigilancia ecocardiográfica. Se resalta la importancia de realizar una evaluación ecocardiográfica en pacientes con trombofilia.

( $) 2018$ Sociedad Colombiana de Cardiología y Cirugía Cardiovascular. Publicado por Elsevier España, S.L.U. Este es un artículo Open Access bajo la licencia CC BY-NC-ND (http:// creativecommons.org/licenses/by-nc-nd/4.0/).
\end{abstract}

\section{Hereditary thrombophilia and intracardiac thrombus}

Abstract Thrombophilia is a haematological condition that predisposes to venous and arterial thromboembolic events. It is also a predisposing factor in intracardiac thrombi in paediatrics, and must be suspected if there are no other factors that predispose to thrombosis. The case is presented on a patient with a history cerebral venous thrombosis, who was diagnosed with thrombophilia due to a homozygote G202010A mutation of the prothrombin gene. She developed a right atrial thrombus, which was treated with anticoagulation and echocardiography monitoring. The importance of performing an echocardiography evaluation is highlighted in patients with thrombophilia.

( $) 2018$ Sociedad Colombiana de Cardiología y Cirugía Cardiovascular. Published by Elsevier España, S.L.U. This is an open access article under the CC BY-NC-ND license (http:// creativecommons.org/licenses/by-nc-nd/4.0/).

\footnotetext{
* Autor para correspondencia.

Correo electrónico: jmiguelmex@hotmail.com (J.M. Torres-Martel).
} 


\section{Introducción}

La incidencia de trombosis en el paciente pediátrico se estima en $0,07 / 10.000$. Dentro de las causas está la trombofilia, una condición hematológica de carácter hereditario (genético) o adquirido, que favorece la aparición, persistencia o recurrencia de eventos tromboembólicos arteriales o venosos. Se presenta con eventos de abortos recurrentes, tromboembolia pulmonar, infarto agudo de miocardio, eventos vasculares cerebrales y trombosis venosa profunda. La etiología de la forma heredada se debe a situaciones asociadas a variaciones genéticas únicas o múltiples (mutaciones y/o polimorfismos genéticos) que afectan a proteínas implicadas en el sistema hemostásico entre las que se encuentran la deficiencia de antitrombina III, el déficit o la resistencia a la proteína $C$, el déficit de proteína $S$, la mutación del gen de protrombina (factor II), las mutaciones de la metiltetrahidrofolato reductasa C677T, A1298C, el factor V de Leiden y la protrombina G20210A. Estas dos últimas tienen menos importancia en cuanto al riesgo trombogénico individual, pero son más frecuentes en la población general y adquieren importancia cuando se asocia a un segundo defecto genético o un factor de riesgo adquirido. La mutación G20210A del gen de la protrombina se asocia al incremento de riesgo de trombosis venosa, con una prevalencia en la población general del 1 al $3 \%^{1-6}$.

La trombosis intracardíaca es una condición cardiológica rara en la práctica clínica pediátrica, pero su aparición es una fuente significativa de morbilidad y mortalidad. Se han identificado como factores de riesgo la obesidad, la prematurez, las infecciones sistémicas, las neoplasias malignas, las enfermedades renales, el síndrome de distrés respiratorio, la enfermedad cardíaca congénita, la miocardiopatía dilatada, los catéteres intravasculares, las enfermedades autoinmunes y las condiciones congénitas o adquiridas protrombóticas o los estados de hipercoagulabilidad. Su detección se ha incrementado gracias a la utilización cada vez más frecuente de estudios de imagen ecocardiográfica ${ }^{7,8}$.

Los trombos en la aurícula derecha rara vez tienen complicaciones, pero en caso de presentarse son eventos catastróficos ya que existe la posibilidad de embolia a la circulación pulmonar o de manera paradójica a la circulación sistémica. El tratamiento de estos trombos se considera un reto, especialmente en pacientes asintomáticos con trombos antiguos, debido a que aún no existen guías de manejo en la población pediátrica?.

Se describe el caso de una paciente con antecedente de un evento vascular cerebral, diagnosticada con trombofilia congénita, que presentó un trombo auricular derecho.

\section{Caso}

Paciente femenina, de 2 años 11 meses de edad, con antecedentes de madre de 32 años con eventos recurrentes de trombosis venosa profunda, en manejo con acenocumarina y familiares por rama materna con eventos de trombosis venosa profunda recurrente. Inició a los 17 meses de edad con un cuadro de infección de vías respiratorias superiores, posteriormente con náusea y vómito, por lo cual se sospechó infección gastrointestinal y se dio manejo médico con metoclopramida. Presentó disminución del estado de alerta y movimientos anormales, sospechándose intoxicación por metoclopramida. Sufrió pérdida del estado de alerta y crisis de espasticidad, por lo que hizo una resonancia magnética de cráneo en la que se halló trombosis venosa cerebral del sistema profundo. Se inició manejo con enoxaparina $1 \mathrm{mg} / \mathrm{kg} /$ dosis cada 12 horas y se hospitalizó en terapia intensiva pediátrica durante 12 días. Requirió intubación endotraqueal 6 días y colocación de catéter venoso central. Se realizó un ecocardiograma transtorácico sin alteraciones aparentes. Se inició protocolo de estudio para trombofilia tanto en la madre como en la paciente, encontrándose positivas a la mutación homocigota G202010A del gen de la protrombina (mutación del factor II). Se suspendió la enoxaparina después de 15 meses de tratamiento. En la actualidad se encuentra con manejo con antagonista de la vitamina $\mathrm{K}$ ( $45 \mathrm{mg} /$ semana) y aspirina $5 \mathrm{mg} / \mathrm{kg} /$ día buscando lograr un INR terapéutico (2 a 3,5).

Fue referida al servicio de Cardiología pediátrica para evaluación previo inicio de su programa de rehabilitación neurológica. La madre la refería asintomática cardiovascular, con una clase funcional I de Ross. A la exploración física se detectaron ruidos cardiacos rítmicos, de buena intensidad y frecuencia sin soplos aparentes, s2 sin alteraciones, pulsos adecuados y llenado capilar menor a 3 segundos. Se realizó ecocardiograma transtorácico debido al antecedente de trombosis venosa cerebral y de colocación de catéter intravascular durante su estancia en terapia intensiva, encontrándose una imagen compacta hiperecogénica compatible con un trombo organizado, con diámetro máximo de $12 \times 7 \mathrm{~mm}$, implantado en la valva septal de la válvula tricúspide y con ligera adherencia en el ángulo formado con el tabique auricular, introduciéndose en la sístole al ventrículo derecho, sin generar obstrucción del sistema venoso o de la válvula tricúspide. No existían gradientes ni se encontró cardiopatía estructural (fig. 1).

Se hizo manejo en conjunto con Hematología y Cirugía cardiovascular, considerando que la paciente no era candidata a trombólisis intravenosa ya que se trataba de un trombo antiguo. No se consideró el manejo quirúrgico debido a que la bomba de circulación extracorpórea predispone a trombosis y embolia intraoperatoria, y no está exenta de complicaciones neurológicas (hasta $1,7 \%$ de los pacientes sometidos las presenta) y la paciente tuvo secuelas neurológicas por el evento cerebral vascular previo y riesgo incrementado de trombosis.

\section{Discusión}

La trombosis en la edad pediátrica es infrecuente (5,1/100.000 recién nacidos vivos). La localización intracardiaca es la más común (25-28\%), seguida de la arterial periférica, sistema nervioso central, sistema venoso mayor intraabdominal y venoso periférico. La trombosis intracardíaca espontánea en ausencia de alguna enfermedad cardíaca predisponente es un evento raro. Se ha descrito después de traumatismo cardíaco, enfermedad inflamatoria multisistémica, enfermedad de Behçet y trombofilia ${ }^{10,11}$.

Esta última fue descrita por primera vez en 1956 por Jordan y Nandoff. Se define como la tendencia a desarrollar tromboembolia venosa o arterial, con un sustrato de 

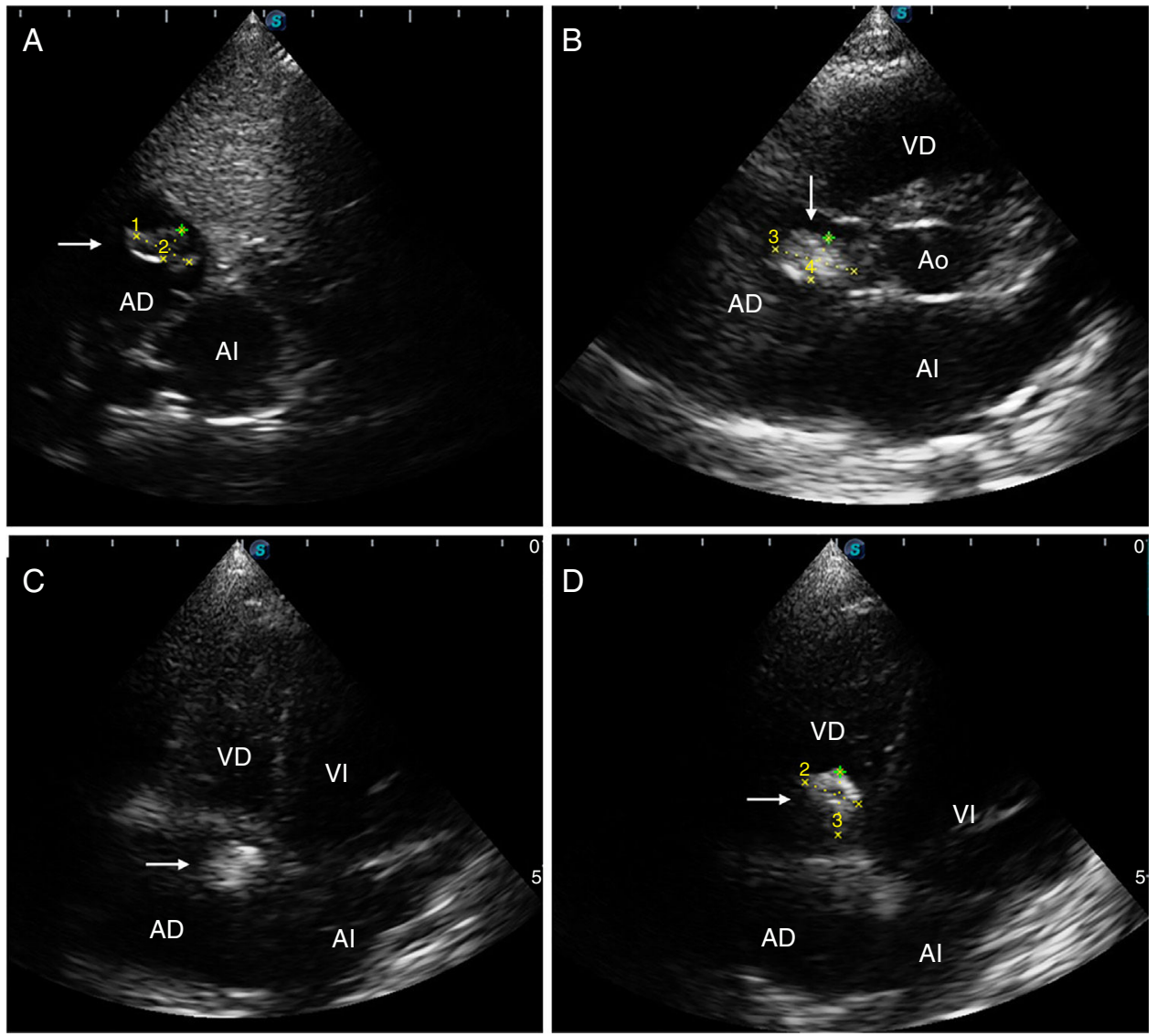

Figura 1 Ecocardiograma transtorácico. A) Imagen subcostal que muestra una imagen hiperecogénica organizada de $12 \mathrm{~mm} x$ $7 \mathrm{~mm}$ en la aurícula derecha sugestiva de trombo cardíaco. B) Imagen de eje corto en la que se observa trombo de $10,5 \times 5,9 \mathrm{~mm}$ adosado a la valva septal de la válvula tricúspide y al ángulo formado con el tabique auricular. C) Imagen de cuatro cámaras en que se aprecia el trombo adherido a la valva septal de la válvula tricúspide y en el ángulo formado con el tabique auricular. D) Imagen de cuatro cámaras que evidencia la apertura valvular tricúspide y el trombo ventricular derecho de 8,6 × 9,4 mm. Ventrículo derecho $(\mathrm{VD})$; ventrículo izquierdo $(\mathrm{VI})$; aurícula derecha $(\mathrm{AD})$; aurícula izquierda (Al); aorta (Ao).

hipercoagulabilidad o alteraciones en la fibrinólisis. Puede ser congénita, adquirida o mixta. Se han descubierto factores de riesgo hereditarios y adquiridos de tromboembolia venosa (tabla 1), que confieren una susceptibilidad variable a la trombosis, generalmente asociada a situaciones de riesgo adquiridas, tales como cirugía, infecciones, inmovilización prolongada, procedimientos intravasculares, terapia hormonal, etc ${ }^{12}$.

Se debe sospechar trombofilia hereditaria en pacientes con tromboembolia venosa recurrente, historia familiar de trombosis venosa, edad menor de 45 años y ausencia de factores de riesgo que expliquen el desarrollo de la trombosis. Las características de los trombos intracardíacos en las trombofilias hereditarias no difieren de las de los trombos intracardíacos vistos en el periodo postinfarto o durante fibrilación auricular. En el caso expuesto, existía historia familiar de trombosis venosa por línea materna, la madre era menor de 45 años (en tratamiento con acenocumarina) y contaba como factor de riesgo para el desarrollo de trombo intracardíaco auricular derecho la colocación de un catéter venoso central durante su estancia en terapia intensiva ${ }^{13}$.

Las características ecocardiográficas de alto riesgo en un trombo en la aurícula derecha incluyen: tamaño mayor de
Tabla 1 Factores de riesgo hereditarios y adquiridos de tromboembolia venosa

\begin{tabular}{|c|c|}
\hline Hereditarios & Adquiridos \\
\hline Factor V de Leiden & Síndrome antifosfolípido \\
\hline $\begin{array}{l}\text { Mutación G202010A de la } \\
\text { protrombina }\end{array}$ & $\begin{array}{l}\text { Resistencia adquirida a la } \\
\text { proteína } C \text { activada }\end{array}$ \\
\hline Déficit de proteína $C$ & Hiperhomocisteinemia \\
\hline Déficit de proteína $S$ & Embarazo y puerperio \\
\hline Déficit de antitrombina & $\begin{array}{l}\text { Uso de anticonceptivos } \\
\text { orales }\end{array}$ \\
\hline Otros & Cáncer \\
\hline $\begin{array}{l}\text { Hiperhomocisteinemia debida } \\
\text { a mutación MTHFR }\end{array}$ & $\begin{array}{l}\text { Cirugía, trauma o } \\
\text { inmovilización }\end{array}$ \\
\hline Disfibrinogenemia & Trombosis previa \\
\hline \multicolumn{2}{|l|}{ Aumento del factor VIII } \\
\hline \multicolumn{2}{|l|}{ Protrombina Yukuhashi } \\
\hline Factor IX Padua & \\
\hline
\end{tabular}

$2 \mathrm{~cm}$, movilidad, forma serpenteante y móvil, pedunculado y manifestación de síntomas. La paciente del caso tenía un trombo de $12 \times 7 \mathrm{~mm}$ sin características ecocardiográficas 
de alto riesgo. En pacientes con trombos auriculares derechos de bajo riesgo en el ecocardiograma, asintomáticos y hemodinámicamente estables, se inicia monitorización continua con estudios de imagen y terapia anticoagulante. Se puede utilizar heparina de bajo peso molecular o heparina no fraccionada, en lugar de warfarina, considerando que las primeras son confiables y tienen mayor margen terapéutico. Su pronóstico generalmente es bueno. Para pacientes sintomáticos o de alto riesgo, se debe considerar cada caso de manera individualizada para direccionar el tratamiento con terapia anticoagulante o trombolítica o trombectomía quirúrgica. El clínico debe hacer un balance del riesgo-beneficio en términos de riesgo de sangrado, mortalidad quirúrgica y pronóstico para decidir la modalidad terapéutica ${ }^{14}$.

En la paciente, el diagnóstico de trombofilia se confirmó con la demostración de la mutación homocigota del gen de protrombina G20210A. La mutación homocigota confiere un riesgo mucho mayor de trombosis comparado con la deficiencia de proteína $\mathrm{C}$ o la mutación homocigota del factor $\mathrm{V}$. No tenía síntomas cardiovasculares al momento de su valoración inicial y sí un trombo que al ecocardiograma no mostró factores de riesgo aparentes, por lo que en conjunto con Hematología y Cirugía cardiovascular se decidió manejo conservador con anticoagulación y vigilancia ecocardiográfica estrecha. A la fecha permanece asintomática desde el punto de vista cardiovascular.

\section{Conclusión}

Existe un riesgo incrementado de trombosis intracardíaca en pacientes con trombofilia hereditaria, de ahí que se deba efectuar una evaluación en esta población, que de igual forma requiere manejo multidisciplinario para un tratamiento exitoso.

\section{Financiación}

Ninguna.

\section{Conflictos de intereses}

Ninguno.

\section{Agradecimientos}

A las Doctoras Lydia Rodríguez Hernández y Aída Mashenka Moreno González por su colaboración en la redacción de este manuscrito.

\section{Bibliografía}

1. Torres V, Saddi V. Systematic review: hereditary thrombophilia associated to pediatric strokes and cerebral palsy. J Pediatr. 2015;91:22-9.

2. Lacayo-Leñero D, Hernández-Hernández D, Valencia-Martínez A, Barrales-Benítez O, Vargas-Ruiz A. Primary thrombophilia in Mexico: a single tertiary referral hospital experience. Blood Coagul Fibrinolysis. 2016;27:920-4.

3. Saini K, Patnaik M, Nagar V, Deshpande A. JIACM. 2005;6:244-7.

4. Girolami A, Berti G, Treleani M, Tasinato V, Girolami B. Congenital thrombophilia and intracardiac thrombosis: probably an underdiagnosed event. Cardiol Res. 2013;4:109-15.

5. Ruiz-Argüelles G, Garcés-Eisele J, Reyes-Núñez V, RamírezCisneros F. Primary thrombophilia in Mexico. II. Factor V G1691A (Leiden), prothrombin G20210A, and methylenetetrahydrofolate reductase $\mathrm{C677T}$ polymorphism in thrombophilic mexican mestizos. Am J Hematol. 2001;66:28-31.

6. Ruiz-Argüelles G, González-Carrillo M, Estrada-Gómez R, Valdés-Tapia P, Parra-Ortega I, Porras-Juárez A. Trombofilia primaria en México. Parte Vl: Falta de asociación estadística entre las condiciones trombofílicas heredadas. Gac Méd Méx. 2007;143:317-22.

7. Ilker Çetin I, Ekici F, Ünal S, Kocabas A, Sahin S, Uysal Yazıcı $M$, et al. Intracardiac thrombus in children: the fine equilibrium between the risk and the benefit. Pediatr Hematol Oncol. 2014;31:481-7.

8. Cabrera-Payne Y, Castillo González D, Rodríguez-Pérez LL, Tejeda-González M, Fonseca Polanco C, Rubio Blanco Y. Mutación G20210A del gen de la protrombina: presentación clínica en dos gestantes. Revista Cubana de Hematología, Inmunología y Hemoterapia V 31. 2015:459-65.

9. Fabi M, Gesuete V, Testa G, Balducci A, Picchio F, Gargiulo G. Calcified thrombus in right atrium: rare but treatable complication of long-term indwelling central venous catheter. Cardiol Res. 2011;2:189-92.

10. Fay K, Maher K, Kogon B. Pediatric intracardiac thrombus: a diagnostic and therapeutic dilemma. Congenit Heart Dis. 2013;8:E157-60.

11. Ohlenforst S, Mueller J, Rox J, Oldenburg J, Pötzsch B. Recurrent intracardiac thrombosis as an unusual manifestation of inherited thrombophilia. Int J Cardiol. 2007; 114:380-1.

12. Martínez-Calle N, Páramo J. Protocolo diagnóstico de trombofilia. Medicine. 2012;11:1377-81.

13. Çinier G, Öz A, Tekkesin A, Hayırogłu M, Keskin M, Avsar Ș. A Young male patient with multiple thromboembolisms associated with factor $v$ Leiden Mutation. Int Heart J. 2016; 57:654-6.

14. Yang J, Williams S, Branda o L, Chan A. Neonatal and childhood right atrial thrombosis: recognition and a risk-stratified treatment approach. Blood Coagulation and Fibrinolysis. 2010;21:301-7. 